\title{
Harder than just hitting a bulls-eye: treatment for hypothyroidism in children might have more than just one target
}

\author{
Camilo Gonzalez-Velazquez $\mathbb{D}^{1} \cdot$ Monica H. Xing $\mathbb{D}^{1} \cdot$ Mark L. Urken $^{1}$
}

Received: 18 February 2020 / Accepted: 3 March 2020 / Published online: 11 March 2020

(c) Springer Science+Business Media, LLC, part of Springer Nature 2020

We read with interest the Cannavo et al. article, which evaluates levothyroxine requirements in children with acquired hypothyroidism [1]. While the reported results are interesting, we discuss two areas of the study that may detract from the clinical significance and applicability of their findings.

The authors find that children with central hypothyroidism secondary to pituitary tumor treatment require a higher dose of levothyroxine to achieve euthyroidism in comparison to children with hypothyroidism due to Hashimoto's thyroiditis. In this study, patients with Hashimoto's thyroiditis were diagnosed based on both thyroid ultrasonography characteristics and thyroid antibodies (TPOAbs/TGAbs) while patients with central hypothyroidism were not diagnosed in the same manner. While we understand that abnormal findings are not expected in these cases, coexisting thyroid autoimmune disease in children with central nervous system tumors or postradiotherapy central hypothyroidism $(\mathrm{CeH})$ cannot be excluded and could skew the results of this study.

Furthermore, the use of FT4 values and TSH values to define treatment target dose in $\mathrm{CeH}$ and $\mathrm{HT}$ groups, respectively, inherently renders these values incomparable. Therefore, the use of differing parameters to form a ROC curve to establish a cutoff value of LT4 dosage is imprecise. In addition, part of the treatment goal was to achieve "clinical euthyroidism," which was not explicitly defined within the study. Clinical hypothyroidism, is known to include an assortment of signs and symptoms that do not have an established direct relationship with LT4 dose or TSH levels. Specifically, in the pediatric population, there are no concrete measures that can definitively attribute slowed growth and development to hypothyroidism rather than organic disorders [2]. Of note, hypothyroidism symptoms may also be

Monica H. Xing

mxing@thancfoundation.org

1 Thyroid, Head and Neck Cancer (THANC) Foundation, 10 Union Square East, Suite 5B, New York, NY 10003, USA underreported by pediatric patients who lack the capacity to thoroughly communicate them.

Some thyroid hormone responses in central peripheral tissues may actually be a consequence of T3 levels, which the current study does not account for. Studies in adults have suggested that isolated low T3 values might be responsible for hypothyroidism symptoms or metabolic changes [3]. Lastly, it is well known that $\mathrm{T} 3$ hormone levels play an important role in the hypothalamic-pituitary-thyroid feedback loop, which should be accounted for when defining treatment goals in patients with hypothyroidism of different etiologies.

Though we understand the conclusions made by Cannavo et al. [1] to be unsubstantiated by the results presented in the article, we readily acknowledge the merit of this research as the framework for future research on this subject.

Acknowledgements The authors acknowledge the Mount Sinai Health System for its support of this research.

\section{Compliance with ethical standards}

Conflict of interest M.L.U. is the Medical Advisor of the THANC Foundation.

Publisher's note Springer Nature remains neutral with regard to jurisdictional claims in published maps and institutional affiliations.

\section{References}

1. L. Cannavo, T. Aversa, D. Corica, G. Pepe, G.B. Pajno, A. Alibrandi, F. De Luca, M. Wasniewska, In children with acquired hypothyroidism levothyroxine requirements may be significantly conditioned by the etiology of thyroid failure. Endocrine 67(1), 252-255 (2020). https://doi.org/10.1007/s12020-019-01965-2

2. A.J. Wassner, Pediatric hypothyroidism: diagnosis and treatment. Paediatr. Drugs 19(4), 291-301 (2017). https://doi.org/10.1007/ s40272-017-0238-0

3. R. Bunevicius, G. Kazanavicius, R. Zalinkevicius, A.J. Prange Jr., Effects of thyroxine as compared with thyroxine plus triiodothyronine in patients with hypothyroidism. N. Engl. J. Med 340(6), 424-429 (1999). https://doi.org/10.1056/NEJM199902113400603 